Cahiers d'études africaines

$240 \mid 2020$

Soudan : identités en tension

\title{
Exposition 120 vaches
}

\section{Alice Franck et Katarzyna Grabska}

\section{(2) OpenEdition}

Journals

Édition électronique

URL : https://journals.openedition.org/etudesafricaines/32883

DOI : 10.4000/etudesafricaines.32883

ISSN : $1777-5353$

\section{Éditeur}

Éditions de l'EHESS

\section{Édition imprimée}

Date de publication : 2 décembre 2020

Pagination : 1023-1026

ISSN : 0008-0055

\section{Référence électronique}

Alice Franck et Katarzyna Grabska, «Exposition 120 vaches », Cahiers d'études africaines [En ligne], 240 | 2020, mis en ligne le 02 décembre 2020, consulté le 05 janvier 2023. URL : http://

journals.openedition.org/etudesafricaines/32883 ; DOI : https://doi.org/10.4000/etudesafricaines. 32883

Ce document a été généré automatiquement le 5 janvier 2023

Tous droits réservés 


\title{
Exposition 120 vaches
}

\author{
Alice Franck et Katarzyna Grabska
}

\section{RÉFÉRENCE}

Exposition 120 vaches. - Le CAP, Centre d'arts plastiques, Saint-Fons, France (23/02/2019-30/03/2020).

1 En février 2019, l'exposition 120 vaches s'ouvrait au CAP (Centre d'Arts Plastiques) de Saint-Fons, en banlieue lyonnaise, où elle a duré jusqu'en fin mars 2019. L'ambition de cette exposition artistique et documentaire était de comprendre et de donner à voir la révolte de figures féminines à travers les questions de discrimination envers les femmes de toutes origines culturelles et sociales en convoquant des pratiques documentaires (vidéo), théâtrales (théâtre forum) et performatives (artistes contemporains). À l'origine et au centre de cette exposition, une proposition de SOS Sud-Soudan et d'Aliza Monchy, fondatrice et présidente de l'association qui milite pour les droits des femmes sud-soudanaises et plus particulièrement contre les pratiques de mariage forcé. 120 vaches reflète les expériences de femmes et d'hommes sud-soudanais vivant au Soudan et au Soudan du Sud, mais aussi dans la diaspora, qui sont confrontés aux coutumes de mariage de défunt. Il s'agit de mariages qui interviennent dans des cas de veuvage, où la femme est donnée/confiée à un «mari de substitution », choisi par la famille du défunt, dont le rôle sera d'en protéger la famille et de poursuivre la filiation, mais également, dans des cas où un garçon est décédé avant d'avoir été marié et d'avoir eu des enfants, pour lui donner une filiation.

2 L'exposition souhaite ouvrir une réflexion sur les hommes et les femmes résistant.e.s, révolté.e.s, face aux contraintes des normes sociales et à la pluralité des violences dont ils sont l'objet ${ }^{1}$. Nous avons été impliquées dans ce projet en tant que chercheuses - une anthropologue et une géographe -, travaillant depuis deux décennies au Soudan et au Sud-Soudan sur les processus de marginalisation sociale et les déplacements forcés, dans une perspective d'analyse de genre. Nous avons mené une enquête, rythmée par des entretiens, à Lyon avec Aliza Monchy, et à Khartoum avec sa famille, 
complétés par une série d'entretiens menés à Lyon auprès de membres de la diaspora sud-soudanaise de Lyon et de Finlande, invités pour l'occasion. Parallèlement, une session de deux jours de théâtre forum a été organisée rassemblant, en plus des personnes déjà évoquées, des artistes comme l'illustratrice Malvina Barra, et l'artiste Fabien Pinaroli. Ces sessions de théâtre ont été animées par la Compagnie L'Archipel et filmées par l'association Gnama Gnama Productions, qui milite pour la création et le développement de projets audiovisuels. L'ensemble du projet a été mis en œuvre par les curateurs Fabien Pinaroli et Nicolas Audureau. Plus d'une quinzaine de personnes (artistes, chercheurs, protagonistes) ont participé à la réalisation de cette exposition.

3 Résultat d'une co-production entre chercheuses, artistes, activistes et protagonistes engagés dans le projet, cette exposition interroge le type de savoirs produit par la création, et la manière dont une œuvre artistique peut être lue dans le cadre d'un processus de recherche. Elle fait appel à différentes modalités artistiques et d'autres médiums que le texte scientifique pour donner à voir et à comprendre de manière sensible différentes expériences de vie : les paroles et récits y sont représentés dans les planches de bandes dessinées) (Malvina Barra) et les vidéos documentaires (Gnama Gnama Productions), lesquelles restituent également la performance des artistes et des protagonistes lors de sessions de théâtre forum. À cela s'ajoute enfin le travail de terrain des chercheuses à Lyon et à Khartoum. Quel type de savoir cette expérience, qui se situe entre les pratiques académiques et artistiques, nous a-t-elle permis d'appréhender et de co-produire? Comment les différentes parties du projet (recherche, théâtre, bande dessinée, vidéos, etc.) se sont-elles articulées en une réflexion collective?

4 Notre participation en tant que chercheuses au montage de l'exposition mais aussi au vernissage, ou encore au débat public auquel le vernissage a donné lieu, nous a permis de considérer les dialogues interdisciplinaires qui façonnent les pratiques artistiques actuelles et comment celles-ci ont travaillé le « contemporain " à partir de thèmes tels que l'altérité, l'identité, la construction du lieu et la violence de genre. De cette façon, nous avons participé avec les artistes et les protagonistes de l'exposition à nourrir un débat, à l'intersection entre l'art (arts visuels, performance) et les sciences sociales ${ }^{3}$, sur les connaissances et les savoirs concernant les violences basées sur le genre et, plus spécifiquement, les mariages de défunts sud-soudanais. Les discussions et les négociations autour des interprétations des pratiques et des normes sociales qui sont intervenues au sein de l'équipe au cours du projet nous ont permis d'appréhender la nature située des savoirs ${ }^{4}$ et d'expérimenter un processus de construction des savoirs.

5 Contactées à l'origine en tant que chercheuses, nous apparaissions aux yeux des autres membres du projet et de la protagoniste centrale, Aliza, comme des « sachants » ayant pour rôle de « traduire » et de rendre explicite aux yeux de tous la révolte d'Aliza et son indignation à l'égard de pratiques de mariage largement méconnues du grand public. Pourtant, l'élaboration de l'exposition nous a conduites à sortir de ce rôle et a fait émerger une réflexion collective dans laquelle les matériaux de recherche ont été modelés par les artistes. Les entretiens ont en effet été écoutés par les artistes vidéastes qui les filmaient et qui s'en sont emparés, sélectionnant des passages, en coupant d'autres, pour donner à voir ce qu'ils souhaitaient mettre en relief. Nous avons aussi beaucoup échangé avec Malvina Barra, l'illustratrice, lui confiant nos photos de terrain pour qu'elle s'imprègne d'atmosphères de là-bas. Ces nombreux allers-retours entre «terrain» de recherche et expériences de vies individuelles (d'Aliza et d'autres 
personnes) ont ensuite été travaillés collectivement comme un matériel artistique. Cette expérience collective a sans doute été plus sensible encore dans le cadre du théâtre forum où chacun a dû sortir de son rôle, et ne pas se contenter d'être spectateur ou observateur, mais s'engager et lâcher-prise pour devenir acteur et donner à voir une réalité complexe et en mouvement. La répétition des scènes et des scénarios avec les changements de rôles des participants éclaire les savoirs situés en permettant à chaque acteur d'« expérimenter " la vie des autres ${ }^{5}$. La manière dont le public s'empare de l'histoire et devient actif dans la production de scénarios alternatifs engage un processus pendant lequel public et acteurs (y compris les chercheurs) expérimentent, démontrent, analysent et transforment en parallèle la réalité.

Dans cette expérience partagée, les différences culturelles et linguistiques s'expriment, se confrontent et s'enchevêtrent. Les manières de penser le mariage, l'amour, le couple, la famille ont ainsi fait l'objet de nombreuses négociations, témoignant de la pluralité des points de vue, et des parcours en lien avec la diversité des normes sociales. Ces échanges ont permis de dépasser collectivement l'idée hâtive que certaines pratiques étaient intrinsèquement injustes et d'éclairer la complexité de l'articulation entre normes sociales, contexte situé et expériences individuelles et la manière dont cette articulation conduit ou non à la révolte et à la transformation d'un ordre politique et social. Ils ont ainsi nourri notre compréhension des savoirs situés et ont permis de coproduire une version complexe des rapports de pouvoirs qui se nouent dans les coutumes de mariage du défunt. Dans ce sens, cette approche au croisement de l'art et des sciences nous a offert une autre perspective sur cette thématique des violences faites aux femmes. Elle a révélé des rapports de pouvoirs enchâssés, en soulignant par exemple la complicité des femmes dans ces violences. Dans le processus de production de l'exposition, nous avons découvert comment l'art et la co-production des savoirs peuvent nous amener à transformer des idées, « à produire une nouvelle perception du monde et donc à créer un engagement pour sa transformation $»^{6}$.

\section{NOTES}

1. Les travaux de deux autres artistes féministes, Camille Ducellier et Tabita Rezaire, complétaient l'exposition, mais ne sont pas traités dans cet article, voir: <https:// lecap-saintfons.com/archives-2019/>.

2. A. S CHNEIDER (DIR.), Alternative Art and Anthropology: Global Encounters, Londres, Bloomsbury, 2017.

3. Ibid.

4. D. HARAWAY, «Situated Knowledges: The Science Question in Feminism and the Privilege of Partial Perspective », Feminist Studies, 14 (3), 1988, pp. 575-599.

5. L'idée du théâtre forum est née dans les années 1970 au Brésil avec le théâtre des opprimés (theatre of the oppressed) élaboré par Augusto Boal.

6. J. RANCIÈRE, Le partage du sensible : esthétique et politique, Paris, La Fabrique, 2000. 


\section{AUTEURS}

\section{ALICE FRANCK}

Université Paris 1 Panthéon-Sorbonne, France

\section{KATARZYNA GRABSKA}

Peace Research Institute, Oslo, Norvège; Institut d'Ethnologie, Université de Neuchâtel, Suisse. 